\title{
Up-regulation of SLC9A9 Promotes Cancer Progression and Is Involved in Poor Prognosis in Colorectal Cancer
}

\author{
MASAMI UEDA ${ }^{1,2}$, TOMOHIRO IGUCHI ${ }^{1}$, TAKAAKI MASUDA ${ }^{1}$, HISATERU KOMATSU $^{1,2}$, SHO NAMBARA $^{1}$, \\ SHOTARO SAKIMURA ${ }^{1}$, HIDENARI HIRATA ${ }^{1}$, RYUTARO UCHI ${ }^{1}$, HIDETOSHI EGUCHI $^{1}$, SHUHEI ITO ${ }^{1}$, \\ KEISHI SUGIMACHI ${ }^{1,3}$, TSUNEKAZU MIZUSHIMA ${ }^{2}$, YUICHIRO DOKI ${ }^{2}$, MASAKI MORI ${ }^{2}$ and KOSHI MIMORI ${ }^{1}$ \\ ${ }^{1}$ Department of Surgery, Kyushu University Beppu Hospital, Beppu, Japan; \\ ${ }^{2}$ Department of Gastroenterological Surgery, Graduate School of Medicine, Osaka University, Suita, Japan; \\ ${ }^{3}$ Fukuoka City Hospital, Surgery, Fukuoka, Japan
}

\begin{abstract}
Background/Aim: SLC9A9 plays an oncogenic role in esophageal squamous carcinoma and glioblastoma. Herein, we showed an oncogenic function of SLC9A9 in colorectal cancer (CRC). Materials and Methods: We examined SLC9A9 expression in CRC specimens by immunohistochemistry. In CRC tissues, the relationship between SLC9A9 expression and clinicopathological factors was further elucidated by quantitative real-time polymerase chain reaction ( $q R T-P C R)$ and gene set enrichment analysis (GSEA). In vitro, we performed knockdown and overexpression experiments. Results: SLC9A9 was overexpressed in CRC specimens. In clinicopathological analysis of our cohort, high SLC9A9 expression increased liver metastasis and was correlated with worse prognoses in two cohorts. A significantly positive relationship between SLC9A9 and EGFR was revealed. While knockdown of SLC9A9 suppressed proliferation and anchorageindependent growth, up-regulation of SLC9A9 promoted proliferation and anchorage-independent growth in vitro. Conclusion: SLC9A9 has an oncogenic function by being related to EGFR signaling, suggesting SLC9A9 may be a novel prognostic indicator and a therapeutic target in CRC.
\end{abstract}

Colorectal cancer (CRC) is one of the most common cancers worldwide and the second leading cause of cancer-related deaths in developed countries, with the majority of deaths being attributed to distant metastasis (1). While the 5-year survival rate of patients with local CRC is about $80 \%$, that of patients with cancer metastasis is still only $10-20 \%$ (2).

Correspondence to: Prof. Koshi Mimori, MD, PhD, Department of Surgery, Kyushu University, Beppu Hospital, 4546 Tsurumihara, Beppu, Oita, 874-0838, Japan. Tel: +81 977271650, Fax: +81 977271651,e-mail: kmimori@beppu.kyushu-u.ac.jp

Key Words: SLC9A9, colorectal cancer, prognosis, EGFR.
Therefore, it is necessary to identify a novel biomarker for cancer progression and metastasis, one that could also be a useful therapeutic target in CRC.

The SLC9A9 gene, also called NHE9, belongs to the $\mathrm{Na}^{+} / \mathrm{H}^{+}$ exchanger superfamily. It localizes on recycling endosomes and maintains homeostasis by electroneutral exchange of protons for $\mathrm{Na}^{+}$through membranes (3). It has been reported that loss of function mutations in SLC9A9 is involved with autism $(4,5)$.

Recently, several studies noted that the SLC9A9 gene contributed to malignant diseases. High expression levels of SLC9A9 are associated with a poor prognosis and cancer progression in esophageal squamous cell carcinoma (6-8). In glioblastoma, SLC9A9 is up-regulated and drives stemness properties, proliferation and invasive growth of glioblastoma cells by activating the EGFR signaling pathway (9).

The role of the SLC9A9 gene has been elusive in CRC. We hypothesized that SLC9A9 might promote the progression of CRC. In the current study, we examined the clinical attribution of SLC9A9 in CRC patients and whether $S L C 9 A 9$ promotes cancer progression.

\section{Materials and Methods}

Patients and samples. Cancerous tissues from a total of 140 CRC patients were obtained during surgery. These samples were collected and used in accordance with the institutional ethical guidelines of Kyushu University after obtaining written informed consent from each patient. All patients underwent resection of the primary tumor at Kyushu University Hospital and affiliated Hospitals between 1992 and 2002. Adjuvant chemotherapy was not performed for any patient of Union for International Cancer Control (UICC) stage III. All patients were clearly identified as having CRC based on clinicopathological findings. The median and mean follow-up were 35.6 and 45.0 months, respectively. Resected tissues were immediately cut and stored in RNAlater (Ambion, Austin, TX, USA), frozen in liquid nitrogen and kept at $-80^{\circ} \mathrm{C}$ until RNA extraction.

Immunohistochemical analysis. CRC tissues were surgically removed, embedded in paraffin and sectioned to a thickness of 5 microns. The 
sections were then stained with hematoxylin and eosin (HE) for histological analysis. Immunohistochemical analysis was applied to determine the expression of SLC9A9. A monoclonal rabbit antiSLC9A9 antibody (ab167157; 1:200; Abcam, Cambridge, UK) was used as the primary antibody.

CRC cell lines. Human CRC cell lines (COLO205, Caco-2, SW480 and HCT116) were provided by the Japanese Cancer Research Bank (Tokyo, Japan). Cell lines were maintained in Dulbecco's modified Eagle's media, RPMI-1640 or Minimum Essential Media supplemented with $10 \%$ fetal bovine serum and antibiotics. All cells were cultured as monolayers at $37^{\circ} \mathrm{C}$ in a humidified atmosphere containing $5 \% \mathrm{CO}_{2}$.

SLC9A9 mRNA interference. SLC9A9-specific siRNA (silencer predesigned siRNA) was purchased from Ambion. The siRNA oligomer was diluted with Opti-MEM I Medium (Invitrogen, Carlsbad, CA, USA). The diluted siRNA oligomer was mixed with Lipofectamine RNAiMAX (Invitrogen) and incubated for $15 \mathrm{~min}$ at room temperature. Diluted logarithmic growth phase CRC cells without antibiotics were seeded at $5 \times 10^{5}$ per $2 \mathrm{ml}$ in 6-well flat-bottom microtiter plates or $5 \times 10^{3}$ cells per $100 \mu \mathrm{l}$ in 96-well flat-bottom microtiter plates. The cells were incubated in a humidified atmosphere $\left(37^{\circ} \mathrm{C}\right.$ and $\left.5 \% \mathrm{CO}_{2}\right)$. The assay was performed after a 72 -h incubation.

Establishment of stable SLC9A9-transfected CRC cells. To generate SLC9A9 expression lentiviral vectors, we amplified the insert (fulllength human NM_173653.3) by polymerase chain reaction (PCR) from human reference cDNA. Lentiviruses were produced by transient transfection of HEK293T cells with pCMV-VSV-G-RSVRev, pCAG-HIVgp and either CSII-CMV-SLC9A9 or CSII-CMVMCS (empty) plasmid DNAs (5'-BamH1 and 3'-Hpa1 sites) plus Lipofectamine 2000 (Invitrogen) following the manufacturer's protocol. Enhanced SLC9A9 expression was confirmed by quantitative RT-PCR and Western blot analysis.

$R N A$ preparation and reverse transcription $(R T)-P C R$. Total RNA from frozen tissue specimens and CRC cell lines was extracted using ISOGEN (Nippon Gene, Tokyo, Japan). cDNA was synthesized from $8 \mu \mathrm{g}$ total RNA with M-MLV reverse transcriptase (Invitrogen).

Quantitative real-time $P C R \quad(q R T-P C R)$ Gene-specific oligonucleotide primers were designed for PCR. The following primers were used: SLC9A9: 5'-CCATGTTGACTTGGCTTCAG-3' (sense) and 5'-GAGGGGTCCTCCTTCAGATT-3' (antisense); glyceraldehyde-3-phosphate dehydrogenase (GAPDH): 5'TTGGTATCGTGGAAGGACTCA-3' (sense) and 5'-TGTCATCA TATTTGGCAGGTT-3' (antisense); EGFR: 5'-TTCCTCCC AGTGCCTGAA-3' (sense) and 5'-GGGTTCAGAGGCTGA TTGTG-3' (antisense). PCR amplification was performed in a LightCycler 480 instrument (Roche Applied Science, Basel, Switzerland) using the LightCycler 480 Probes Master kit (Roche Applied Science), according to the manufacturer's protocol. The relative expression levels of these genes were obtained by normalizing the amount of mRNA to that of GAPDH mRNA as an endogenous control in each sample.

Immunoblotting analysis. Total protein was extracted from CRC cell lines. Aliquots of total protein $(40 \mu \mathrm{g})$ were electrophoresed on $10 \%$ polyacrylamide gels and then electroblotted on nitrocellulose membranes using Trans-Blot Transfer Medium (Bio-Rad Laboratories, Hercules, CA, USA) at 0.4 A for $120 \mathrm{~min}$. SLC9A9 protein was detected by using rabbit monoclonal antibodies (ab167157, 1:2,000; Abcam). Each protein level was normalized to the level of $\beta$-actin protein, which was detected by using monoclonal antibodies (Cytoskeleton, Denver, CO, USA) at a $1: 1,000$ dilution.

MTT assay. CRC cell proliferation was assayed by the 3-(4, 5dimethylthiazol-2-yl)-2, 5-diphenyltetrazolium bromide (MTT) assay. Cells were cultured in 96-well flat-bottom microtiter plates and an MTT assay kit from Roche Applied Science was used to evaluate cell proliferation.

Sphere formation assay. The sphere formation assay was performed to assess anchorage-independent growth. Cell suspensions of each cell line were plated on ultra-low adherent $10-\mathrm{cm}$ dishes (Corning, NY, USA) at $5 \times 10^{4}$ cells per dish in sphere formation medium (1:1 DMEM/F12 medium supplemented with epidermal growth factor (EGF; R\&D Systems, Minneapolis, MN, USA) and B-27 (Invitrogen)). After 7 days, the spheres were counted using the ImageQuant LAS 4000 Mini system (GE Healthcare, Buckinghamshire, UK).

Gene set enrichment analysis (GSEA). The associations between SLC9A9 expression and previously annotated gene expression signatures were analyzed by applying GSEA (10). We acquired CRC expression profiles from the National Center for Biotechnology Information Gene Expression Omnibus (GEO) database (accession codes GSE21815), reported by us, and analyzed these expression profiles using GSEA, as previously described $(11,12)$.

Meta-analysis. We obtained CRC expression profiles and information of survival rates from the Cancer Genome Atlas database (TCGA) containing 593 samples and analyzed the association between SLC9A9 expression and probability of survival rates (13).

Statistical analysis. Data from qRT-PCR analyses were analyzed using JMP 5 software (JMP, Cary, NC, USA). The relationships between SLC9A9 expression and clinicopathological factors were analyzed using the Student's $t$-test, chi-square test and analysis of variance (ANOVA). Overall survival (OS) curves were plotted using the Kaplan-Meier method measured from the day of surgery, while the log-rank test was applied for comparison. According to SLC9A9 expression level, cases were divided into two groups by the minimum $p$-value approach, which is a comprehensive method to find the optimal risk separation cut-off point in continuous gene expression measurements (14).

\section{Results}

CRC tissues expression of SLC9A9. We investigated the expression of SLC9A9 in CRC tissues and their adjacent normal colon tissues obtained from 6 patients and liver metastasis tissue with CRC using immunohistochemical analysis. The analysis revealed that SLC9A9 was largely positive in cancerous tissues (Figure 1A). SLC9A9 expression was primarily seen in the cytoplasm, on the cell 

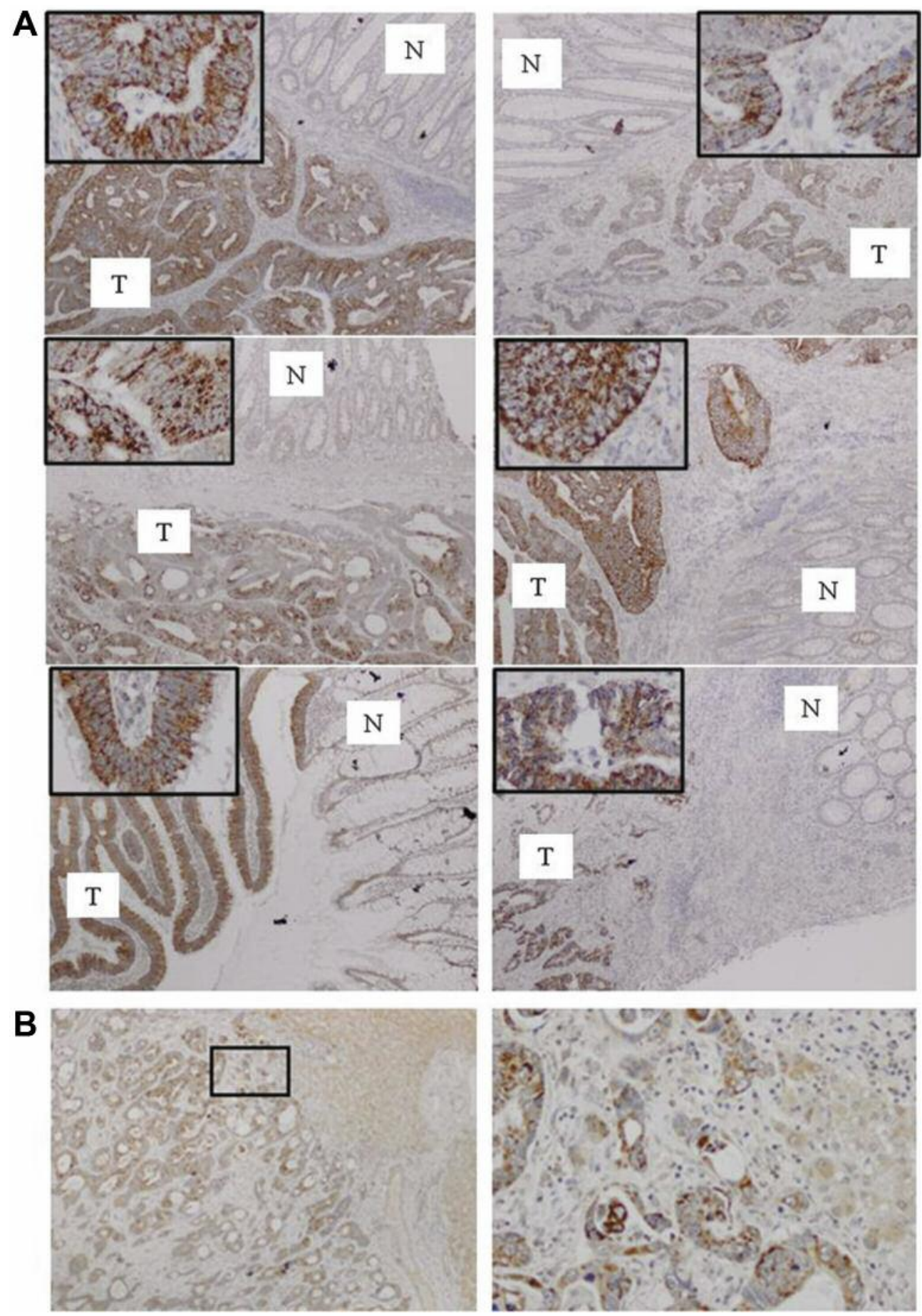

Figure 1. Images of immunohistochemical analysis for SLC9A9 in 6 CRC tissues and one liver metastasis tissue. A. The SLC9A9 expression in tumor tissues was higher than that in normal tissues in 6 samples. Most staining occurred in cytoplasm and cell membrane of cancer cells; original magnification, 40x, 200x. T: Tumor tissue. N: normal tissue. B. Immunohistochemical analysis of liver metastasis tissues revealed strong SLC9A9 expression in cytoplasm and cell membrane of cancer cells; original magnification, 40× (left), 200× (right).

membrane or both. However, the level of SLC9A9 expression in normal epithelium was far lower than that of cancerous tissues. Furthermore, We also showed that
SLC9A9 was up-regulated in CRC cells of liver metastasis (Figure 1B). The results supported that SLC9A9 might contribute to malignancies in human CRC. 
SLC9A9 expression and clinicopathological characteristics. We divided the $140 \mathrm{CRC}$ patients of our clinical cohort into a high-SLC9A9 group $(\mathrm{n}=65)$ and a low-SLC9A9 group $(\mathrm{n}=75)$, according to the $S L C 9 A 9$ expression level in tumor tissues. Clinicopathological factors were analyzed in the high- and low-SLC9A9 expression groups (Table I). The high-SLC9A9 group had significantly more liver metastases than the lowSLC9A9 group. With regard to overall survival (OS), the high$S L C 9 A 9$ group had a significantly poorer prognosis than the low-SLC9A9 group ( $p=0.0042$; Figure 2A). On multivariate analysis, high-SLC $9 A 9$ expression was an independent prognostic factor for poorer OS (hazard ratio $(\mathrm{HR})=1.60 ; 95 \%$ confidence interval $(\mathrm{CI})=1.05-2.55 ; p=0.027$; Table II). Moreover, we analyzed OS from a public data set of 593 CRC cases (TCGA). In accordance with our results, the OS of the high-SLC9A9 group was significantly poorer than that of the low-SLC9A9 group ( $p=0.0055$; Figure $2 \mathrm{~B})$.

Expression of SLC9A9 correlated with EGFR signaling. In order to investigate the oncogenic signaling that is associated with SLC9A9, we applied GSEA to CRC cases (GSE21815). Our analysis demonstrated that SLC $9 A 9$ was positively associated with EGFR signaling in the CRC cohort (Figure $3 \mathrm{~A})$. In addition, we also confirmed a significantly positive association between SLC9A9 and EGFR in our clinical cohort of 140 CRC samples by qRT-PCR. These findings supported that SLC9A9 might promote progression of CRC in conjunction with EGFR signaling.

Inhibition of SLC9A9 expression with SLC9A9 SiRNA in $C R C$. To investigate the effect of inhibition of SLC9A9 in CRC, we performed knockdown experiments in cell lines (COLO205 and Caco-2) whose levels of SLC9A9 mRNA were relatively high. The protein levels of SLC9A9 in cell lines transfected with SLC9A9 siRNA were significantly lower than those with negative control siRNA (Figure 4A). The transfected cells were used to examine whether tumor proliferation and anchorage-independent growth were repressed. It was demonstrated that cell proliferation (Figure 4B) and sphere formation capacities (Figure 4C and D) were significantly lower in SLC9A9 siRNA-transfected CRC cells than in negative control siRNA-transfected CRC cells.

Overexpression of SLC9A9 enhanced the growth of CRC cell lines. To explore the influence of up-regulation of SLC9A9 on CRC cells, we performed overexpression experiments in SW480 and HCT116 cells, which exhibit relatively low expression of SLC9A9. We confirmed by western blotting that SLC9A9 protein expression was higher in cells transfected with SLC9A9 cDNA than in mock-cells (Figure 5A). Next, we performed MTT and sphere formation assays to assess the influence on tumor proliferation and anchorage-independent growth. Overexpression of SLC9A9 significantly promoted
Table I. Relationships between SLC9A9 expression and clinicopathological factors.

\begin{tabular}{|c|c|c|c|}
\hline \multirow[t]{2}{*}{ Factors } & \multicolumn{2}{|c|}{ SLC9A9/GAPDH } & \multirow[b]{2}{*}{$p$-Value } \\
\hline & $\begin{array}{l}\text { High expression } \\
\quad \mathrm{n}=65\end{array}$ & $\begin{array}{c}\text { Low expression } \\
n=75\end{array}$ & \\
\hline \multicolumn{4}{|l|}{ Age } \\
\hline$\leq 65$ & 26 & 28 & 0.74 \\
\hline$>65$ & 39 & 47 & \\
\hline \multicolumn{4}{|l|}{ Gender } \\
\hline Male & 45 & 43 & 0.14 \\
\hline Female & 20 & 32 & \\
\hline \multicolumn{4}{|l|}{ Histology } \\
\hline Well, Moderate & 58 & 72 & 0.11 \\
\hline Others & 7 & 3 & \\
\hline \multicolumn{4}{|l|}{ Size $^{\mathrm{a}}$} \\
\hline$<5 \mathrm{~cm}$ & 34 & 40 & 0.92 \\
\hline$\geq 5 \mathrm{~cm}$ & 29 & 33 & \\
\hline \multicolumn{4}{|l|}{ Tumor location } \\
\hline Right colon & 19 & 27 & 0.24 \\
\hline Left colon & 20 & 14 & \\
\hline Rectum & 26 & 34 & \\
\hline \multicolumn{4}{|l|}{ UICC TNM stage } \\
\hline $\mathrm{I} / \mathrm{II}$ & 31 & 38 & 0.72 \\
\hline III/IV & 34 & 37 & \\
\hline \multicolumn{4}{|l|}{$\mathrm{T}$ category } \\
\hline 1,2 & 17 & 29 & 0.11 \\
\hline 3,4 & 48 & 46 & \\
\hline \multicolumn{4}{|c|}{ Lymph node metastasis } \\
\hline Absent & 33 & 41 & 0.64 \\
\hline Present & 32 & 34 & \\
\hline \multicolumn{4}{|l|}{ Liver metastasis } \\
\hline Absent & 56 & 72 & $0.035^{\mathrm{b}}$ \\
\hline Present & 9 & 3 & \\
\hline \multicolumn{4}{|l|}{ Lymphatic invasion } \\
\hline Absent & 32 & 46 & 0.15 \\
\hline Present & 33 & 29 & \\
\hline \multicolumn{4}{|l|}{ Venous invasion } \\
\hline Absent & 51 & 59 & 0.97 \\
\hline Present & 14 & 16 & \\
\hline
\end{tabular}

The 140 colorectal cancer cases were divided into 2 groups according to SLC9A9 mRNA expression levels in cancer tissues. High SLC9A9 expression was significantly associated with liver metastasis $(p=0.035)$. UICC, Union for International Cancer Control; TNM, tumor, node, metastasis. anformation of 4 cases is unknown. bStatistically significant $(p<0.05)$.

cell proliferation (Figure 5B) and sphere formation of cancer cells (Figure 5C and D). These results suggested that SLC9A9 might play a role in promoting the progression of CRC.

\section{Discussion}

In the current study, we report the oncogenic role of SLC9A9 in CRC. First, by immunohistochemical staining, we ensured that $S L C 9 A 9$ was up-regulated in primary CRC 

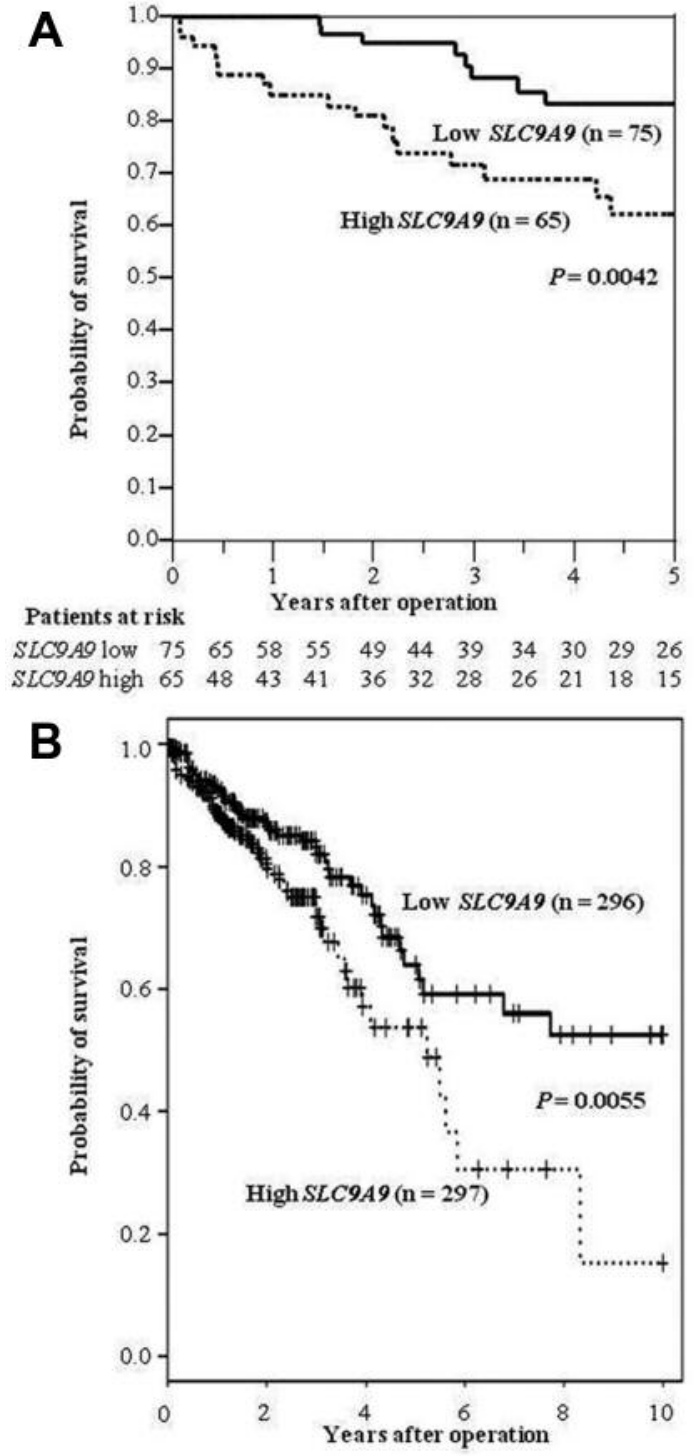

Figure 2. Kaplan-Meier survival curves for CRC patients as a function of SLC9A9 expression level. A. A total of 140 CRC cases were classified into two groups according to the SLC9A9 expression level. The survival rate for patients in the high-expression group $(n=65)$ was significantly lower than that for patients in the low-expression group $(n=75)$ ( $p=0.0042)$. B. Kaplan-Meier overall survival curves for 593 patients with CRC in the public database of TCGA. The survival rate for patients in the high-expression group $(n=297)$ was significantly lower than that for patients in the low-expression group $(n=296)(p=0.0055)$.

tumors compared to the corresponding normal tissues. In clinical samples, high level of SLC9A9 in CRC tissues was an independent prognostic indicator for OS and significantly involved with liver metastasis in our cohorts, which was supported by the TCGA public database. Moreover, analysis of qRT-PCR in our cohort and GSEA showed a concordant relationship between $E G F R$ and $S L C 9 A 9$ expression.

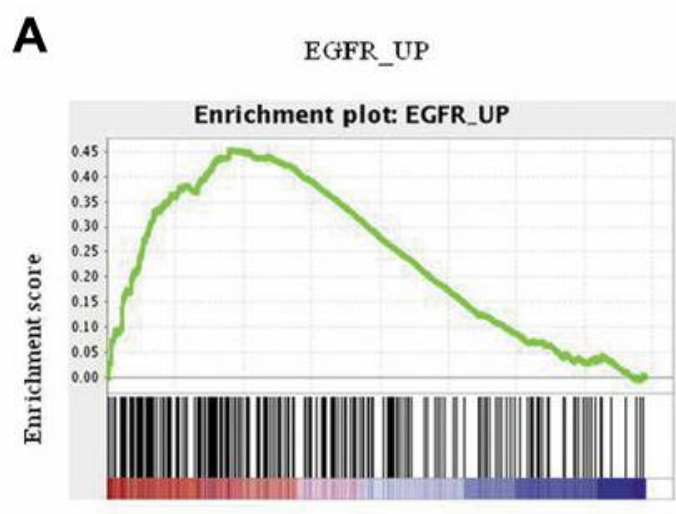

Normalized enrichment $s c o r e=1.6413633$ p-value $=0.025$ q-value $=0.049$

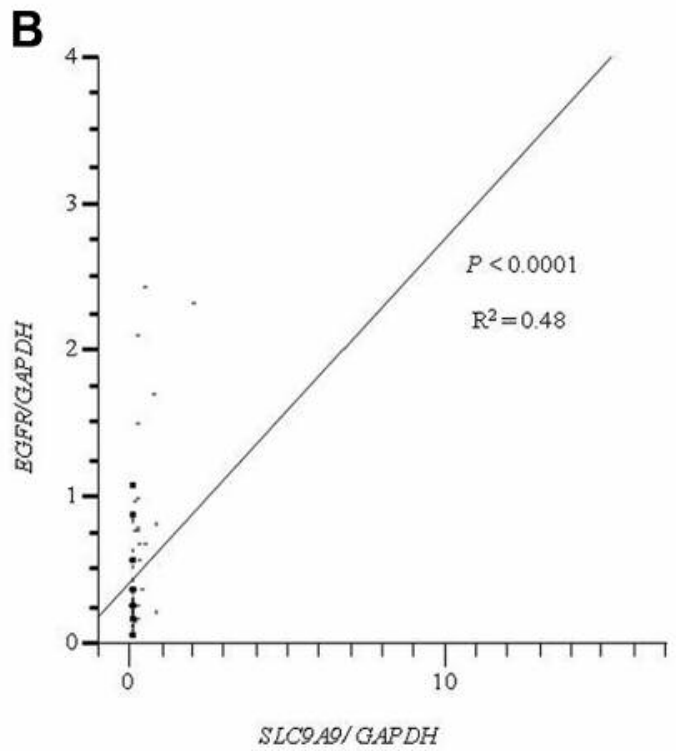

Figure 3. The positive correlation between SLC9A9 and EGFR. A. GSEA of inter-related SLC9A9 and EGFR expression using reference gene sets in a CRC data set. B. Correlations between EGFR and SLC9A9 expression levels were analyzed by $q R T-P C R$ in 140 CRC samples.

EGFR plays a central role in the development of diverse epithelial tumors and provides key signals for tumor progression in CRC (15-17). Since monoclonal antibodies, such as cetuximab and panitumumab, can provide CRC patients with significant clinical benefits (18-21), SLC9A9 might be an important biological molecule, in CRC, that is closely associated with EGFR signaling. High expression of EGFR is reported to have a strong correlation with liver metastasis (22), which also agrees with our clinical data.

Consistent with the above, we showed that SLC9A9 acts as an oncogene in CRC. Specifically, in vitro SLC9A9 
A

COLO205
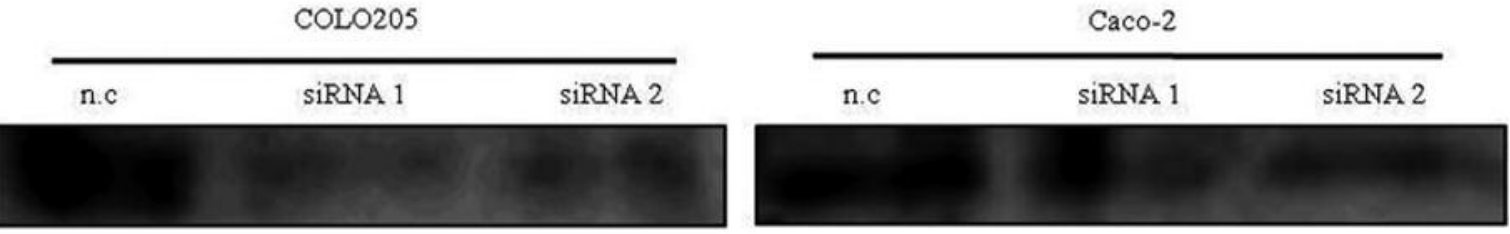

$\beta$-actin
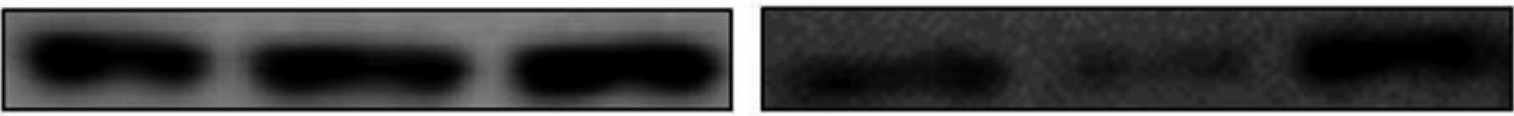

B

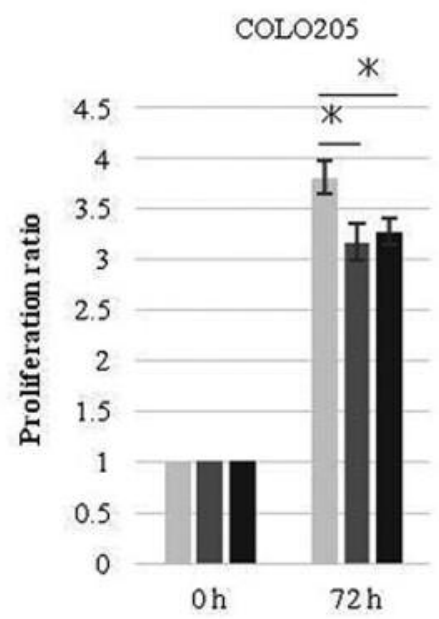

Caco-2

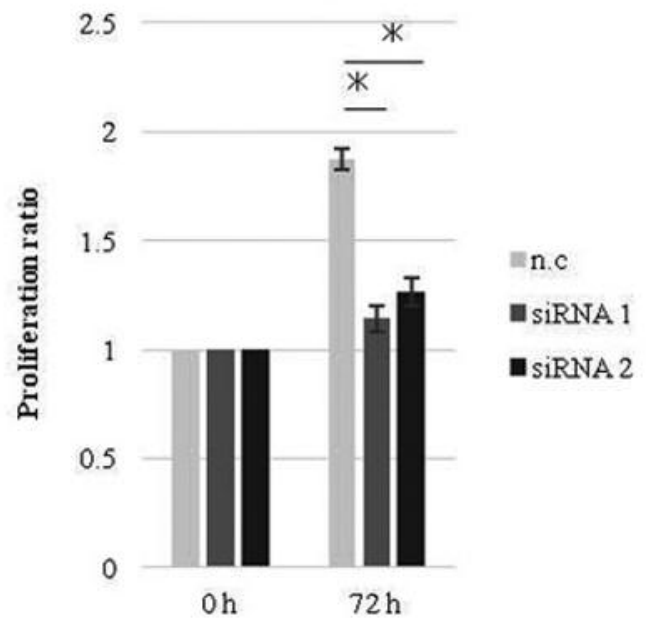

C
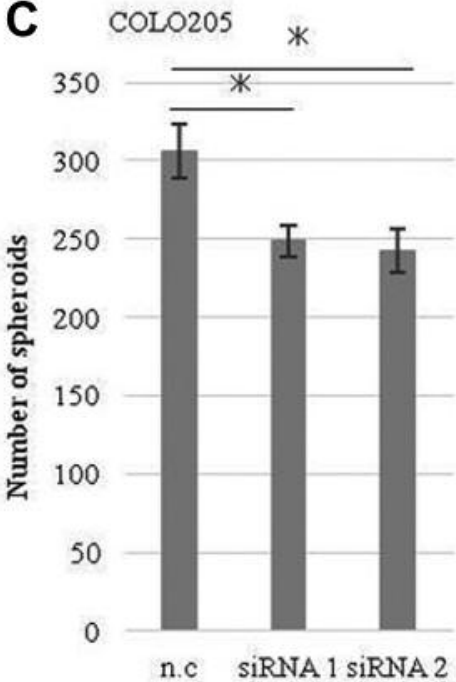

D

COLO205
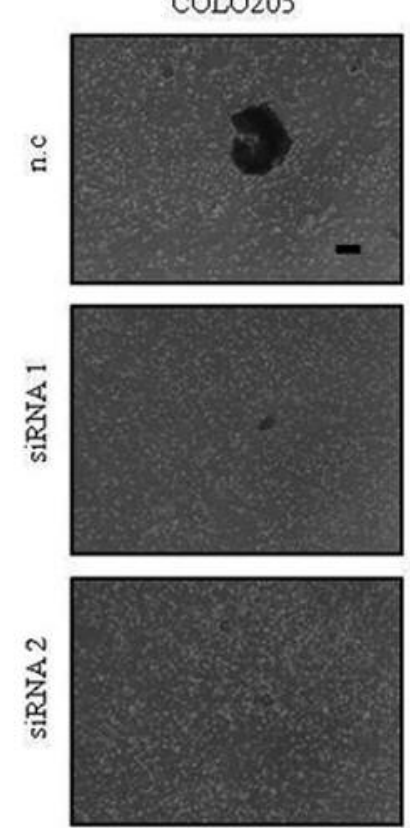

Caco-2

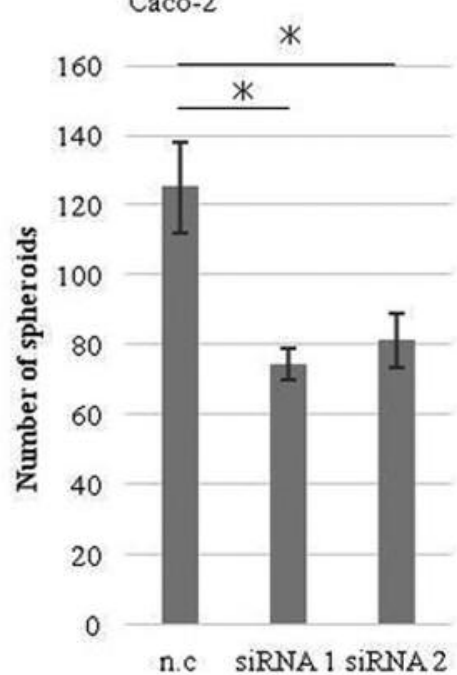

Caco-2
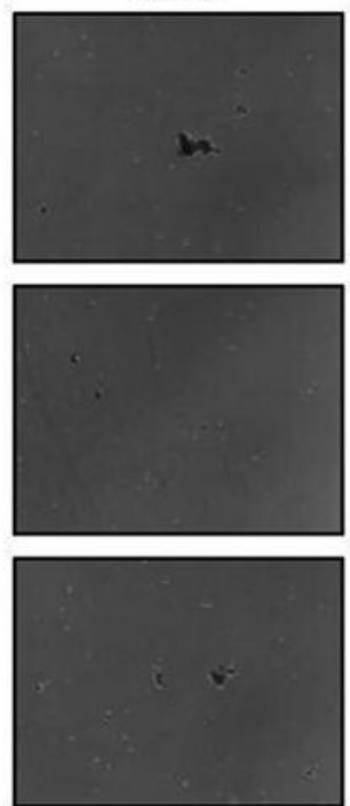

Figure 4. Knockdown of SLC9A9 inhibited the proliferation and sphere formation of human CRC cells. A. Knockdown of SLC9A9 was confirmed by western blotting in COLO205 and Caco-2 cells. B. Knockdown of SLC9A9 inhibited the cell proliferation of both COLO205 and Caco-2 cells. $* p<0.05$. C. Knockdown of SLC9A9 repressed sphere formation of both COLO205 and Caco-2 cells. * $p<0.05 . D$. Representative images of sphere formation are shown. Scale bar, $100 \mu \mathrm{m}$ (Lower). 
A
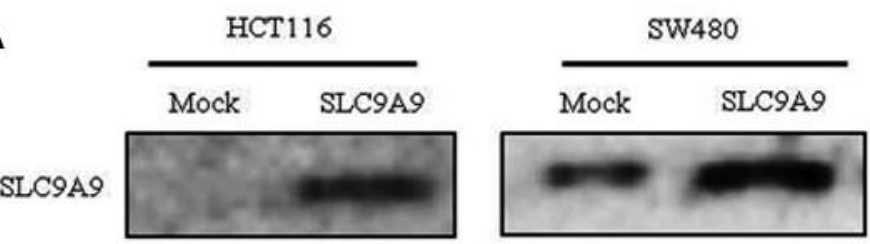

$\beta$-actin
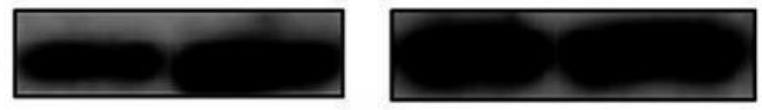

B

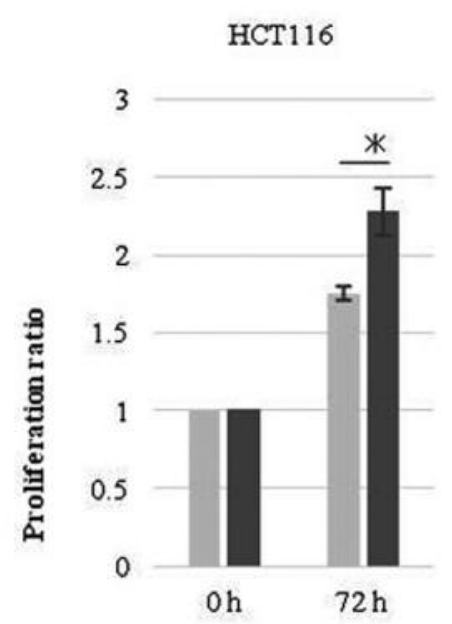

SW480

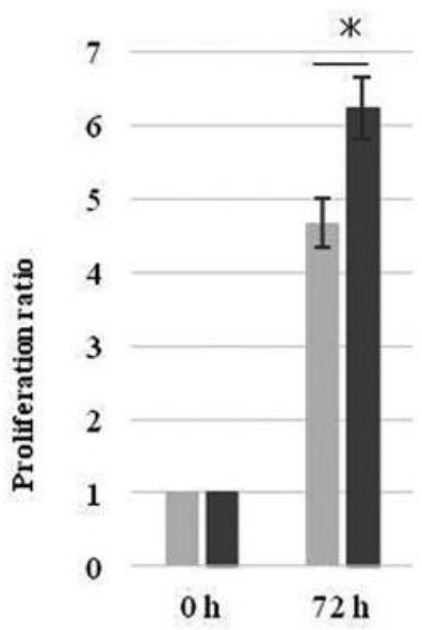

C

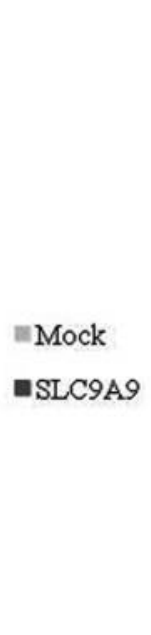

HCT 116

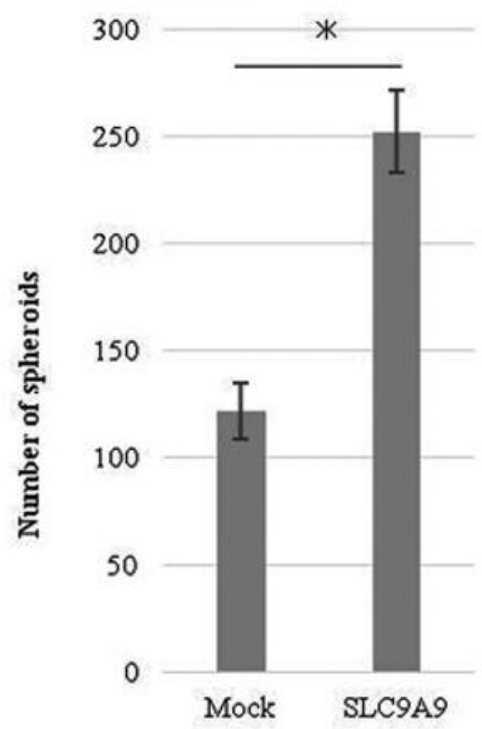

D

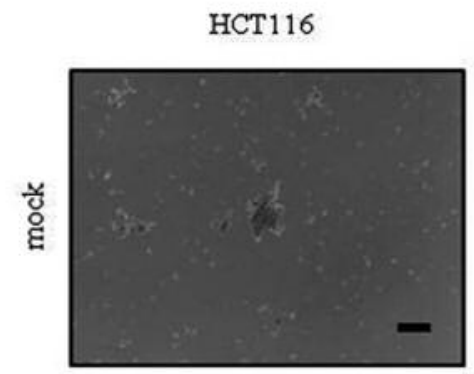

=Mock

-SLC9AS

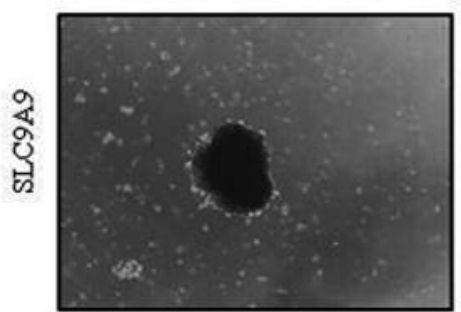

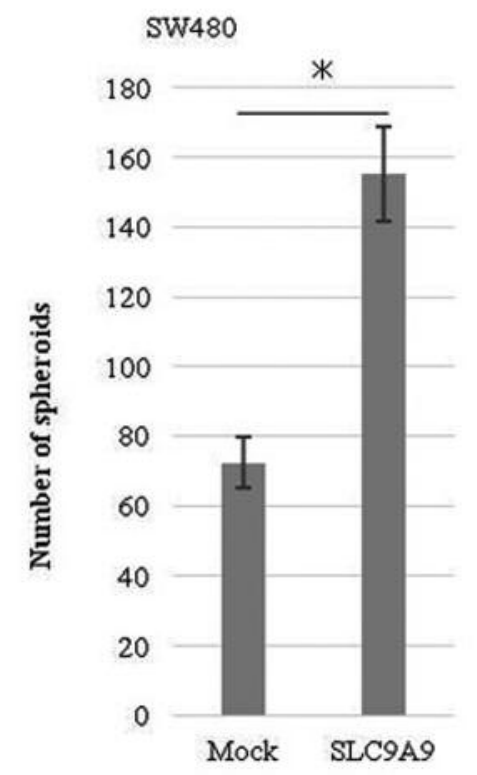

SW480
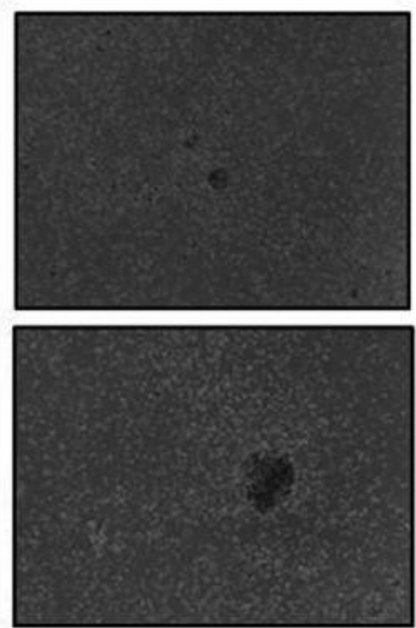

Figure 5. Overexpression of SLC9A9 increased cell proliferation and sphere formation by human CRC cells. A. SLC9A9 was stably enhanced in HCT116 and SW480 cells. B. Overexpression of SLC9A9 enhanced the cell proliferation of both HCT116 and SW480 cells. *p<0.05. C. Overexpression of SLC9A9 increased sphere formation by both HCT116 and SW480 cells. ${ }^{*} p<0.05$. D. Representative images of sphere formation are shown. Scale bar, $100 \mu \mathrm{m}$ (Lower). 
Table II. Univariate and multivariate analysis for overall survival (Cox proportional hazards regression model).

\begin{tabular}{|c|c|c|c|c|c|c|}
\hline \multirow[b]{2}{*}{ Factors } & \multicolumn{3}{|c|}{ Univariate analysis } & \multicolumn{3}{|c|}{ Multivariate analysis } \\
\hline & $\mathrm{RR}$ & $95 \% \mathrm{CI}$ & $p$-Value & $\mathrm{RR}$ & $95 \% \mathrm{CI}$ & $p$-Value \\
\hline UICC T factor (I II/III IV) & 2.79 & $1.52-6.98$ & $<0.001^{*}$ & 1.42 & $0.68-3.74$ & 0.36 \\
\hline Lymphatic invasion (absent/present) & 1.75 & $1.17-2.69$ & $0.0058^{*}$ & 1.27 & $0.78-2.12$ & 0.66 \\
\hline Venous invasion (absent/present) & 2.15 & $1.44-3.21$ & $<0.001 *$ & 2.02 & $1.25-3.31$ & $0.0036^{*}$ \\
\hline Liver metastasis (absent/present) & 3.55 & $2.25-5.39$ & $<0.001 *$ & 2.69 & $1.60-4.51$ & $<0.001 *$ \\
\hline Lymph node metastasis (absent/present) & 2.16 & $1.40-3.59$ & $<0.001 *$ & 1.28 & $0.77-2.22$ & 0.33 \\
\hline Histology (Well, Moderate/others) & 2.4 & $1.29-3.92$ & $0.0088^{*}$ & 1.89 & $0.96-3.38$ & 0.061 \\
\hline SLC9A9 expression (low/high) & 1.69 & $1.13-2.65$ & $0.01 *$ & 1.6 & $1.05-2.55$ & $0.027 *$ \\
\hline
\end{tabular}

$* p<0.05$. RR, Relative risk; CI, confidence interval; UICC, Union for International Cancer Control.

overexpression enhanced tumor cell proliferation and anchorage-independent growth of CRC cells. Kondapalli et al. demonstrated that SLC9A9 can activate EGFR signaling pathways in glioblastoma (9) Generally, the majority of endocytosed EGFR is delivered to lysosomes and degraded (23). However, increased SLC9A9 diverts endocytosed EGFR trafficking away from lysosomes and then transfers endocytosed EGFR to the cell membrane for recycling. This process up-regulates EGFR expression on the membrane of glioblastoma cells and activates downstream signaling of EGFR. This report strongly supports our data, considering that SLC9A9 induced cancer progression in CRC.

We revealed that SLC9A9 was up-regulated in CRC tissues compared to corresponding normal tissues by immunohistochemical analysis. However, it is not clear how SLC9A9 expression is regulated. A previous study demonstrated that in hypermutated CRC, SLC9A9 is one of the frequent targets of mutation (13). It is suggested that mutation of SLC9A9 may lead to gain-of-function, which may up-regulate protein level of SLC9A9 in CRC. Hereafter, genetic and epigenetic mechanism(s) regulating SLC9A9 must also be investigated.

In conclusion, the function of SLC9A9 is closely associated with EGFR pathways and can promote progression of CRC. SLC9A9 may be a prognostic indicator and a useful therapeutic target in CRC. However, further studies are necessary to disclose the mechanisms through which SLC9A9 exerts its oncogenic effects in CRC.

\section{Conflicts of Interest}

The Authors declare no conflicts of interest.

\section{Acknowledgements}

This research used the super-computing resource provided by Human Genome Center, the Institute of Medical Science, the University of Tokyo (http://sc.hgc.jp/shirokane.html). We thank K. Oda, M. Kasagi, S. Kono, M. Aoyagi and T. Kawano for their excellent technical assistance. This work was supported in part by the following grants and foundations: Japan Society for the Promotion of Science (JSPS) Grant-in-Aid for Science Research (grant nos. 25430111, 25461953, 25861199, and 25861200); Japan Science and Technology Agency (JSTA) A-step (grant no. AS242Z03987P); and the Founding Program for Next Generation World-leading Researchers (grant no. LS094).

\section{References}

1 Washington MK: Colorectal carcinoma: Selected issues in pathologic examination and staging and determination of prognostic factors. Arch Pathol Lab Med 132: 1600-1607, 2008.

2 O'Connell JB, Maggard MA and Ko CY: Colon cancer survival rates with the new American Joint Committee on Cancer sixth edition staging. J Natl Cancer Inst 96: 1420-1425, 2004.

3 Orlowski $\mathrm{J}$ and Grinstein S: Diversity of the mammalian sodium/proton exchanger SLC9 gene family. Pflugers Arch 447: 549-565, 2004.

4 Kondapalli KC, Hack A, Schushan M, Landau M, Ben-Tal N and Rao R: Functional evaluation of autism-associated mutations in NHE9. Nat Commun 4: 2510, 2013.

5 Zhang-James Y, Middleton FA, Sagvolden T and Faraone SV: Differential expression of SLC9A9 and interacting molecules in the hippocampus of rat models for attention deficit/hyperactivity disorder. Dev Neurosci 34: 218-227, 2012.

$6 \mathrm{Ng} \mathrm{D}, \mathrm{Hu} \mathrm{N}, \mathrm{Hu}$ Y, Wang C, Giffen C, Tang ZZ, Han XY, Yang HH, Lee MP, Goldstein AM and Taylor PR: Replication of a genome-wide case-control study of esophageal squamous cell carcinoma. Int J Cancer 123: 1610-1615, 2008.

7 Chen J, Wen J, Zheng Y, Yang H, Luo K, Liu Q, Hu R, Tan Z, Huang $\mathrm{Q}$ and $\mathrm{Fu} \mathrm{J}$ : Prognostic significance of SLC9A9 in patients with resectable esophageal squamous cell carcinoma. Tumour Biol 36: 6797-6803, 2015.

8 Chen J, Yang H, Wen J, Luo K, Liu Q, Huang Y, Zheng Y, Tan $\mathrm{Z}$, Huang $\mathrm{Q}$ and $\mathrm{Fu} \mathrm{J}$ : NHE9 induces chemoradiotherapy resistance in esophageal squamous cell carcinoma by upregulating the Src/Akt/beta-catenin pathway and $\mathrm{Bcl}-2$ expression. Oncotarget 6: 12405-12420, 2015.

9 Kondapalli KC, Llongueras JP, Capilla-González V, Prasad H, Hack A, Smith C, Guerrero-Cázares H, Quiñones-Hinojosa A 
and Rao R: A leak pathway for luminal protons in endosomes drives oncogenic signalling in glioblastoma. Nat Commun 6: 6289, 2015.

10 Subramanian A, Tamayo P, Mootha VK, Mukherjee S, Ebert BL, Gillette MA, Paulovich A, Pomeroy SL, Golub TR, Lander ES and Mesirov JP: Gene set enrichment analysis: Aa knowledgebased approach for interpreting genome-wide expression profiles. Proc Natl Acad Sci USA 102: 15545-15550, 2005.

11 Kogo R, Shimamura T, Mimori K, Kawahara K, Imoto S, Sudo T, Tanaka F, Shibata K, Suzuki A, Komune S, Miyano S and Mori M: Long noncoding RNA HOTAIR regulates polycombdependent chromatin modification and is associated with poor prognosis in colorectal cancers. Cancer Res 71: 6320-6326, 2011.

12 Yokobori T, Iinuma H, Shimamura T, Imoto S, Sugimachi K, Ishii H, Iwatsuki M, Ota D, Ohkuma M, Iwaya T, Nishida N, Kogo R, Sudo T, Tanaka F, Shibata K, Toh H, Sato T, Barnard GF, Fukagawa T, Yamamoto S, Nakanishi H, Sasaki S, Miyano S, Watanabe T, Kuwano H, Mimori K, Pantel K and Mori M: Plastin3 is a novel marker for circulating tumor cells undergoing the epithelial-mesenchymal transition and is associated with colorectal cancer prognosis. Cancer Res 73: 2059-2069, 2013.

13 Cancer Genome Atlas Network: Comprehensive molecular characterization of human colon and rectal cancer. Nature 487: 330-337, 2012.

14 Mizuno H, Kitada K, Nakai K and Sarai A: PrognoScan: A new database for meta-analysis of the prognostic value of genes. BMC Med Genomics 2: 18, 2009.

15 Messa C, Russo F, Caruso MG and Di Leo A: EGF, TGF-alpha, and EGF-R in human colorectal adenocarcinoma. Acta Oncol 37: 285-289, 1998.

16 Jaszewski R, Levi E, Sochacki P, Frank J, Kucuk O, Axelrod BN and Majumdar AP: Expression of epidermal growth factorreceptor related protein (ERRP) in human colorectal carcinogenesis. Cancer Lett 213: 249-255, 2004.

17 Rego RL, Foster NR, Smyrk TC, Le M, O’Connell MJ, Sargent DJ, Windschitl $\mathrm{H}$ and Sinicrope FA: Prognostic effect of activated EGFR expression in human colon carcinomas: Comparison with EGFR status. Br J Cancer 102: 165-172, 2010.
18 Cunningham D, Humblet Y, Siena S, Khayat D, Bleiberg H, Santoro A, Bets D, Mueser M, Harstrick A, Verslype C, Chau I and Van Cutsem E: Cetuximab monotherapy and cetuximab plus irinotecan in irinotecan-refractory metastatic colorectal cancer. N Engl J Med 351: 337-345, 2004.

19 Van Cutsem E, Peeters M, Siena S, Humblet Y, Hendlisz A, Neyns B, Canon JL, Van Laethem JL, Maurel J, Richardson G, Wolf $M$ and Amado RG: Open-label phase III trial of panitumumab plus best supportive care compared with best supportive care alone in patients with chemotherapy-refractory metastatic colorectal cancer. J Clin Oncol 25: 1658-1664, 2007.

20 Ciardiello $\mathrm{F}$ and Tortora G: EGFR antagonists in cancer treatment. N Engl J Med 358: 1160-1174, 2008.

21 Van Cutsem E, Köhne CH, Hitre E, Zaluski J, Chang Chien CR, Makhson A, D’Haens G, Pintér T, Lim R, Bodoky G, Roh JK, Folprecht G, Ruff P, Stroh C, Tejpar S, Schlichting M, Nippgen $\mathrm{J}$ and Rougier P: Cetuximab and chemotherapy as initial treatment for metastatic colorectal cancer. N Engl J Med 360: 1408-1417, 2009.

22 Goldstein NS and Armin M: Epidermal growth factor receptor immunohistochemical reactivity in patients with American Joint Committee on Cancer Stage IV colon adenocarcinoma: implications for a standardized scoring system. Cancer 92: 13311346, 2001.

23 Opresko LK, Chang CP, Will BH, Burke PM, Gill GN and Wiley HS: Endocytosis and lysosomal targeting of epidermal growth factor receptors are mediated by distinct sequences independent of the tyrosine kinase domain. J Biol Chem 270: 4325-4333, 1995.
Received March 20, 2017

Revised April 1, 2017

Accepted April 3, 2017 